\title{
TRANSPLANTATION
}

\section{Host stem cells boost tolerance of liver grafts}
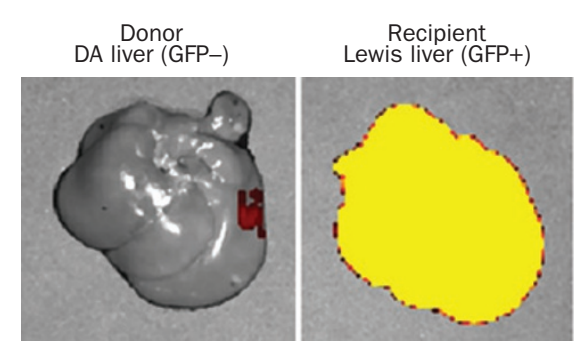

3 months post transplant DA liver (GFP-) into Lewis (GFP+) Epi-flourescence

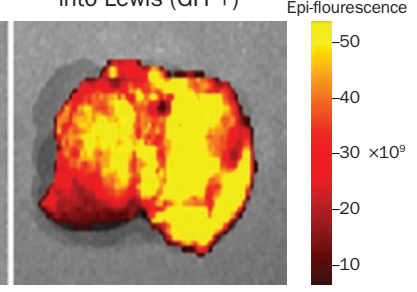

Stem cell repopulation of a small liver allograft in animals treated with plerixafor and low-dose tacrolimus. Transplanted donor liver graft has a high level of fluorescence, indicating that host stem cells have repopulated the graft liver. Abbreviations: DA, Dark Agouti; GFP, green fluorescent protein. Courtesy of Z. Sun, Johns Hopkins University, USA.

Repopulation of donor livers with host stem cells enables long-term acceptance of liver transplants in a rat model that normally strongly rejects organ grafts, according to new research. A combination of low-dose, short-term tacrolimus (to prevent acute rejection) and plerixafor (to mobilize host stem cells to the donor organ) prolonged graft survival and led to the partial conversion of donor livers to a host 'self' phenotype. “This purposeful manipulation of the immune response with low-dose immunosuppression and liberation of stem cells for a very short period after transplantation results in long-term transplant acceptance, without further drug treatment," notes senior author Zhaoli Sun.

As small numbers of host stem cells had been detected in human liver, kidney and heart grafts, Sun and co-workers questioned whether it were possible to manipulate the mobilization of host stem cells such that donor grafts could be 'transformed' to a host phenotype, which would be better tolerated. Two different rats were used: donor livers were taken from Dark Agouti rats and transplanted into transgenic Lewis rats that expressed green fluorescent protein. Host stem cell mobilization could therefore be monitored by measuring levels of fluorescence in the donor liver.

Combination treatment with low-dose tacrolimus and plerixafor for 7 days after transplantation (with a reduced-size liver graft, 50\%) led to long-term graft function and survival ( $>180$ days) in most rats tested (12 of 13), whereas nearly all control rats died within 12 days of transplantation. Interestingly, donor livers in these surviving rats were repopulated with fluorescent host stem cells; moderate green fluorescence was detected within 10 days of transplantation, which became extensive at 3 months. Furthermore, regulatory T cells-which suppress the immune response and help maintain 'self' tolerance-were detected in the blood and liver of the transplanted rats on combination therapy.

This new approach could be the first step towards long-term tolerance of liver transplants without the need for strong immunosuppression, suggest the authors, which would avoid the lifelong treatment with immunosuppressants that is currently needed in transplant recipients. "Our long-term goal is to develop therapeutic strategies for direct clinical application," says Sun, who plans to test these new approaches in large animal preclinical transplantation models and to further characterize the biological mechanisms that underlie the process of stem cell repopulation.

Katrina Ray

Original article Okabayashi, T. et al. Mobilization of host stem cells enables long-term liver transplant acceptance in a strongly rejecting rat strain combination. Am. J. Transplant. doi:10.1111/j.1600-6143.2011.03698.x 